\title{
Medical Image Segmentation Techniques, a Literature Review, and Some Novel Trends
}

\author{
Amira A. Mahmoud ${ }^{1}$, El-Sayed M. El-Rabaie ${ }^{2}$, Taha E. Taha ${ }^{3}$, Adel Elfishawy ${ }^{4}$, \\ Osama Zahran, Fathi E. Abd El-Samie ${ }^{6}$ \\ ${ }^{123456}$ Department of Electronics and Electrical Communications, Faculty of Electronic \\ Engineering, Menoufia University \\ (Received: 20 Sept. 2016 - Accepted: 5 Nov. 2017)
}

\section{Abstract}

Segmentation requires the separation or division of an image into regions of similar properties. Image amplitude is the most basic attribute for image segmentation. Image texture and edges are also useful properties for the segmentation process. There is no standard approach for segmentation of an image; no single theory for image segmentation. Segmentation of an image is usually used to mark and determine boundaries and objects (curves, lines, etc.) in an image. More precisely, image segmentation is the process of labeling of every pixel in the image where pixels having the same properties have the same visual properties and share the same group. The result of segmentation process is a number of regions or segments that cover the whole image, or a number of extracted edges and contours of the image. All pixels in the same region are similar according to some characteristics or properties, such as texture, intensity, or color. In this paper a literature review of the various segmentation methods that are available for medical images is presented. Because of image segmentation importance, a set of image segmentation techniques namely; Thresholding techniques, Clustering techniques, Artificial Neural Networks, Edge based techniques, Region based techniques, Watershed, Graph based and Deformable models have been discussed and compared. The features and requirements of several freely and commercial software tools for image segmentation are clarified. The paper is ended by focusing on the novel trends on the topic. 


\section{Introduction}

Segmentation is the process of partitioning an image into different regions or segments. The aim of segmentation is to divide the image into homogeneous, self-consistent regions, which should correspond to different object in the scene. The process is achieved using only properties of the image. The basic property useful for image segmentation is its amplitude. The other properties such as edges and texture are also useful for segmentation

In medical imaging, these regions often correspond to different tissue classes, organs, or other structures. Medical image segmentation is infected by noise, low contrast, and other imaging artifacts. Although there are many image segmentation techniques, some have been used specially for medical image segmentation.

Image segmentation has an important role in many medical imaging applications, by facilitating the delineation boundaries or edges of organs, structures or other regions of interest. Segmentation techniques are divided into two main categories: discontinuity and similarity. The first category (discontinuity) partitions the image depending on changes in the intensity such as boundaries and edges. The second category (similarity) partitions the image into similar regions according to some criterion.

Qualitative guidelines for good image segmentation have been established as follows:

1. Segmented regions in the image must be homogeneous and uniform according to some characteristics such as texture or gray level.

2. Regions interiors must not contain any small halls and must be simple.

3. Each segmented region should have significantly different values compared to the other adjacent regions with respect to the characteristics on which they are uniform.

4. Accurate boundaries for each segmented region must be simple, accurate and must not be ragged. 
This paper is organized as follows: Section 2, describes the current segmentation techniques for medical image segmentation techniques ended by a comparative study between these techniques. Section 3, concentrates on the novel trends on the topic followed by the conclusions and the more relevant references.

\section{Literature Review on Medical Image Segmentation Techniques}

In the literature, there are a large number of segmentation techniques [1] [9]. Fig. 1 summarizes these techniques where the details for each technique will be discussed.

\subsection{Thresholding}

It is an example of pixel based segmentation algorithm and one of the oldest methods where segmentation is achieved by collecting all pixels having intensity between two thresholding values into the same class. For this segmentation method, pixel amplitude is the most important feature that can be applied in the segmentation process to segment an object from the background. If the desired object is white and the background is black, or vice versa, it is an easy task to set an appropriate threshold to segment this object from its background.

According to how bright or how dark the pixels are, they are labeled as belonging to the object or background. Threshold is selected to differentiate between the dark and the bright levels. The grey-level histogram of an image is studied to determine the image dark and bright regions. The histogram is supposed to have two peaks and the threshold is selected as the point at the minimum in between. To segment more than one object, a multimodal histogram with multiple thresholds could be used. If the mean grey-level in the image varies throughout the image, where the threshold suitable for one region may be unsuitable for the other region, the image can be split into smaller regions in which the mean greylevel is nearly constant and threshold each region as before. 


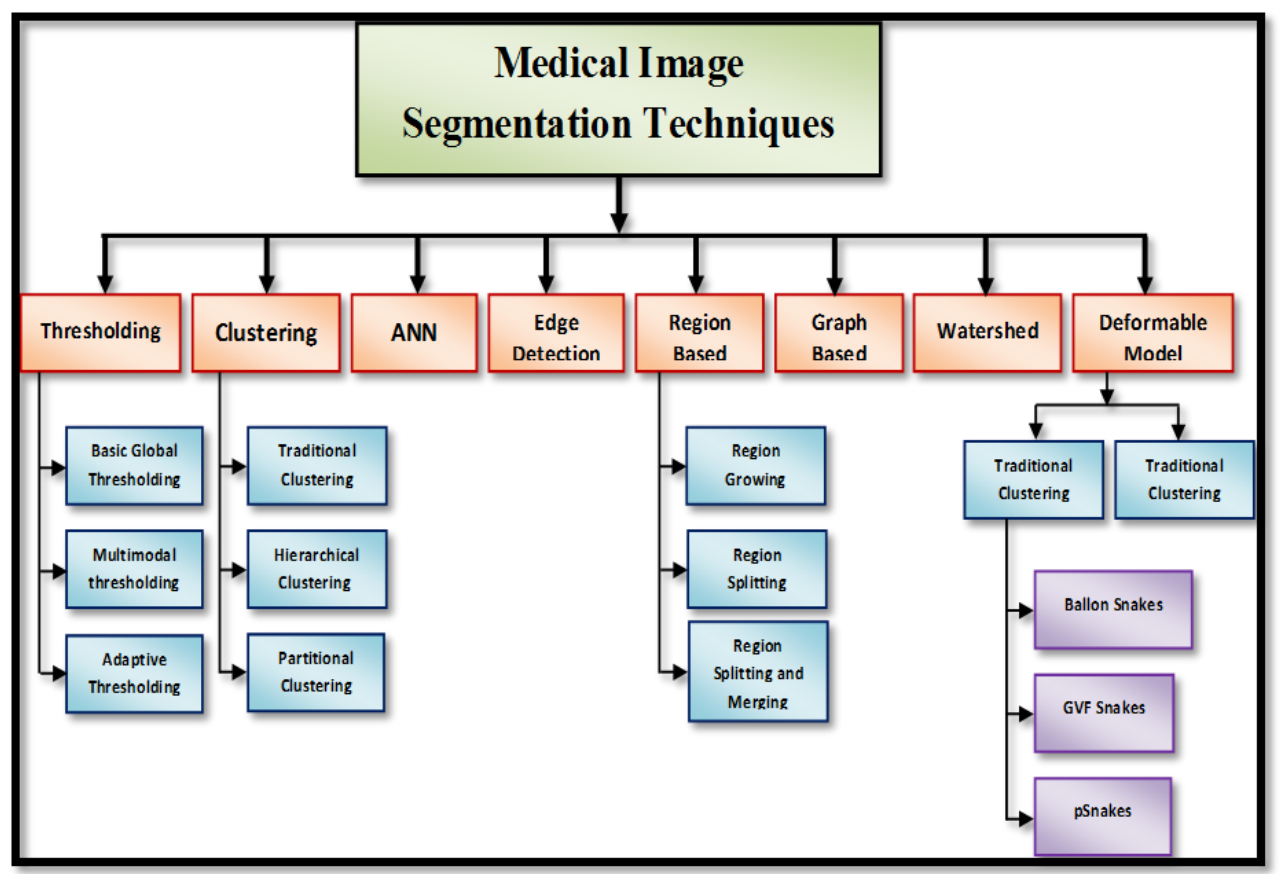

Fig. 1: Medical Image Segmentation Techniques.

Threshold techniques can be divided into two classes: the traditional or the global thresholding and the other is the adaptive thresholding. In the global thresholding, only one single threshold value is used for the entire image. In the adaptive threshold, the threshold value is determined for each pixel to locate it to the object or to the background.

\subsubsection{Basic Global Thresholding}

For a global threshold $\mathrm{T}$, a point $(x, y)$ for which $f(x, y)>T$, is classified as object point otherwise is called background point. In grayscale images (binary converted), the values greater than $\mathrm{T}$ will be one (white) otherwise zero (black).

This produces two groups of pixels, $R_{1}$ which contains pixels with gray level values $>T$ and $R_{2}$ which contains pixels with gray level values $\leq T$. Then the mean (average gray level) values mean $_{1}$ and mean $_{2}$ for pixels in $R_{1 \text { and }} R_{2}$ are computed, and finally compute the 
threshold $T=0.5\left(\right.$ mean $_{1}+$ mean $\left._{2}\right)$. Otsu's method is a famous example of global thresholding.

\subsubsection{Multimodal Thresholding}

The process of determining more than one threshold value is called multithresholding. Multimodal thresholding sets a point $(x, y)$ to one object class if $T_{1}<f(x, y) \leq T_{2}$, to the other object class if $f(x, y)>T_{2}$ and to the background if $f(x, y) \leq T_{1}$.

Thresholding is good choice for medical image (gray-scale images) segmentation, but not popular for remote sensing applications due to high variability of features.

The result of segmentation depends on the identification of the good threshold value. The thresholding techniques have been a popular for many years because of the advantages of simplicity and ease of implementation. But it doesn't work well when dealing with flat images with smooth peaks and valleys.

\subsubsection{Adaptive Thresholding}

The image is divided into regions and the previously mentioned threshold algorithm is applied for each of these regions.

Li Haitao and Li Shengpu [10] proposed an algorithm for image segmentation which is based on the improvement of the gradient histogram. The authors stated that every segmentation algorithm of an image has its own features, drawbacks and aims, so it is important to firstly understand and analysis each image before segmentation process, after that the appropriate algorithm for segmentation of a certain image can be selected and this will conduct to an optimum image segmentation result.

Mohammed J. Islam [11] stated that Computer Vision is the most powerful solution for real time check of capsules in pharmaceutical industry. The author has built a system using edge based segmentation algorithm for quality check. In the first stage, Sobel Edge Detector is used 
to find out edges using noise suppression property. In the next stage, Otsu thresholding algorithm is used to localize the object and the background pixels. Experimental results are compared with Neural Network segmentation technique. Results outperform Neural Network technique according to accuracy and CPU time results.

$\mathrm{Wu}$ Kaihua and Ban Tao [12] have presented a new optimal threshold segmentation method based on entropy criteria and Genetic algorithm in order to improve the image acquisition process in computer vision. The factors taken by them are illumination, light, reflection of light, CCD exposure time and some properties of image histogram. They compare their proposed technique with Otsu algorithm and found that their algorithm is efficient in searching and in finding threshold based segmentation of an image.

Salem Saleh Al-amri, N.V. Kalyankar and Khamitkar S.D [13] proposed a survey on five segmentation techniques based on thresholding of image namely, Mean method, P-tile method, Histogram Dependent Technique (HDT), Edge Maximization Technique (EMT). The five techniques are tested compared with each other in order to choose the one that outperforms the others. The comparative study shows that HDT and EMT techniques are the best techniques for image segmentation threshold techniques.

Weihong Cui Yi Zhang [14] proposed an edge based auto threshold select method to generate multi-scale image segmentation. Band weight and NDVI (Normalized Difference Vegetation Index) is used to calculate edge weight. MST and Edge based Threshold method is used to perform image segmentation. Experiments are performed on multi-scale resolution images, i.e., Quick-bird multispectral images. Results have shown that their method maintain the object information and keep object boundaries while segment the image.

Fengchun Zhang [15] presents a variation model using 4th order PDE with 2nd order PDE for finger vein image de-noising. Midpoint Threshold segmentation technique is used to extract the region of interest accurately. 4th order PDE has reduced the noise very well, whereas 2nd order PDE has approximated the boundaries effectively. It can be observed from experiments that PSNR value of proposed method is increase by $2 \mathrm{~dB}$. Method is compared with threshold based segmentation algorithm and it is 
found that proposed method has segment the real finger vein image accurately.

Anping XU [16] proposed a threshold-based level set approach comprising both threshold based segmentation and Fast Marching Method (FMM) for medical image segmentation. The result of de-noising filter is passed to FMM for segmentation purpose with the help of threshold based level set technique. They implement their method in VC++ and ITK. After the experiment, results have shown that level set method based on threshold results in clearer, accurate and more perfect segmentation, it also avoids from edge leakage and improve the speed of segmentation.

Shiping Zhu [17] proposed a new threshold based edge detection and image segmentation algorithm. They calculate the threshold of each pixel in the image on the basis of its neighboring pixels. They also find the edges of image with the help of proposed algorithm. A threshold of each pixel was set using histogram. PDF is used to isolate the background and threshold of image. They implement their algorithm in Visual C++. Results outperform the Canny Operator results, because it performs edge detection and segmentation simultaneously.

Frank Jiang [18] proposed a new multilevel Threshold-based segmentation technique using PSO and Wavelet mutation. They also proposed a new PSO algorithm which is used in first two steps of algorithm. Then the output of PSO is passed to wavelet mutation operations which performs the mutation operation and update the PSO equations after it. This work will generate optimized threshold and correct segmentation. After comparing their method with HCOCLPSO, they found that it produces optimal threshold as compare to other method. They claim that their algorithm is best for real time applications, e.g., error resilient video application in hostile environment.

\subsection{Clustering}

Clustering is the process of grouping or organizing objects into groups (called clusters) whose members are similar, in some sense, to each other than those in other groups (clusters). It is unsupervised approach that performs segmentation without using training data. Clustering techniques can be categorized into three classes: Traditional clustering, Hierarchical 
clustering and Partitional clustering. Each of these classes is to be explained below.

\subsubsection{Traditional Clustering}

Traditional clustering partition the image into $\mathrm{N}$ segments or clusters. Then combine the best pairs of clusters according to some criterion. When the no. of clusters is reached, the algorithm stops merging regions. In traditional clustering the no. of clusters must be decided before the process begin.

It is simple, and easy to complement but the main problem is to decide the appropriate no of clusters especially at high variability of features.

\subsubsection{Hierarchical Clustering}

Partitional clustering decomposes an image into a set of disjoint clusters then evaluates them by some criterion. Given an image of $\mathrm{N}$ pixels, a partitioning method constructs $\mathrm{K}(\mathrm{N} \geq \mathrm{K})$ partitions of the image, with each partition representing a cluster. That is, it classifies the image into K groups by satisfying the following requirements: (1) each group contains at least one point, and (2) each point belongs to exactly one group.

Advantages of partitional clustering are that it is faster than hierarchical clustering, the no of clusters are fixed, and its concept is simple. One disadvantage of partitional clustering and its initial problem is to determine the no of clusters and the initial centers. Partitional clustering has wide applications in medical imagery combined with other types of artificial neural networks, but is not popular in remote sensing applications.

Nameirakpam Dhanachandra, Khumanthem Manglem and Yambem Jina Chanu[19] proposed an Image Segmentation technique using K-means Clustering Algorithm and Subtractive Clustering Algorithm. K-means clustering algorithm is an unsupervised technique and it is used to segment the interest area from the background. The proposed algorithm consists of partial contrast stretching, subtractive clustering, k-means clustering and median filter. The segmentation process uses K-mean-clustering algorithm 
in parallel with subtractive cluster to locate the initial centroid. The partial contrast stretching algorithm is also used for the quality improvement of original image and the median filter is used for segmented image improvement. Finally, the segmented image is compared to k-means clustering algorithm and results of the proposed method are found to have better performance result.

A. Jose, S. Ravi and M. Sambath[20] proposed a segmentation algorithm for brain tumor making use of K-means clustering and fuzzy C-means algorithm and its area calculation. The process is divided into three stages, the image pre-processing stage, then advanced k-means and fuzzy c-means stage and finally the feature extraction stage. First pre-processing is achieved to improve the image quality using enhancement filter. Then the proposed algorithm is used, K-means followed by Fuzzy C-means to segment the image. The segmented image is then used in extraction stage to extract the feature of the region of interest. MRI image is used in the segmentation process and the extracted tumor size is calculated.

Pallavi P. and Ritesh J. [21] proposed a novel effective approach based on $\mathrm{K}$-means clustering algorithm. The cluster center is generated by the reduction of the final cluster mean square error and there is no large increment in the execution time. The mean square error and the execution time are reduced.

Syoji Kobashi [22] achieved segmentation using scale based fuzzy connected image segmentation and fuzzy object model to the cerebral parenchyma region of brain of new born MRI image. In first step the background region is extracted, then the intensity correction of MRI is performed, and finally scale-base Fuzzy Object Model (FOM) is applied on result image. Evaluation of the results of proposed method isachievedbased on Fast Positive Volume Fraction (FPVF) and Fast Negative Volume Fraction (FVNF). Results from experiment have shown that FOM (Fuzzy object model) acquired the minimum values of FPVF and FVNF.

Anna Fabijańska [23] introduced a new method uses Variance Filter for edge detection in image segmentation process. Their method found the edge position using Variance Filter. Sobel Gradient filter with K-means is also used to extract the edges and compared with the proposed technique. The effect of filtering window size on determining edges is also discussed 
and it is found that if the $9 \times 9$ window is used to extract edges then edge is complete accurately match the shape of object in the image. In case of larger details images, a small filtering window is proffered. Results have shown that their proposed technique outperforms Sobel Edge Detector

M. Yedla, S. R.Pathakota, T. M. Srinivasa[24] proposed Enhancing Kmeans clustering algorithm with improved initial center. The author introduced a novel method to find the initial centroid and provided an effective way to assign a data point to its suitable cluster with reduced complexity. The author proved their proposed approach to have increased accuracy with reduced complexity compared to traditional k-means clustering algorithm. This approach does not need any extra input requirements like thresholding value, but it still initializes the number of clusters $\mathrm{k}$ and how the optimum value of $\mathrm{k}$ is to be determined of the future work.

R. V. Patil [25] claims that if the number of clusters is estimated in accurate manner, K-means image segmentation will provide better results. They proposed a new method based on edge detection to estimate number of clusters. Phase congruency is used to detect the edges. Then these edges are used to find clusters. Threshold and Euclidean distance is used in order to make clusters. K-means is sued to find the final segmentation of image. MATLAB is used to implement the proposed technique. Experiments are performed on nine different images and results shows that number of clusters is accurate and optimal.

L. Yucheng [26] proposed a new image segmentation algorithm using fuzzy morphological. Firstly, morphological opening and closing operations has been used to smooth the image and then, the gradient operations are performed on the resultant image. After comparison with Watershed algorithm and Prewitt methods, the author claimed that their proposed fusion approach solves the over-segmentation problem of Watershed algorithm and it improves the speed as well as it saves the image details.

\subsection{Artificial Neural Networks (ANN)}

A neural network is an artificial simulation of the brain of human which simulates its learning process. The Artificial Neural Network (ANN) is 
simply called a Neural Network or Neural Net. ANN is widely used in order to solve the segmentation problem of medical image. ANN which is based on life simulation, especially the learning of human brain's operations, set up a huge number of parallel nodes. Each one can carry out certain operation. The learning procedure is achieved by the determining the connections between nodes and the weights of the connection. One of the advantages of ANN that it is not dependent on the Probability Density Distribution (PDD) function. It can also prove the results of the segmentation process when the data deviates from the normal situation. ANN can also reduce the complexity requirements of segmentation process.

The disadvantages of ANN can be stated as: it needs three sets of information for efficient segmentation process, also it has difficulties when segmenting image affected by changes of the tissue characteristics so it is not affective for real time applications.

Shohel Ali Ahmed [27] proposed an image texture classification approach based on (ANN). Firstly, image is taken and pre-processed, after that, feature extraction is performed. Finally, the texture classification is performed using ANN classifier so as to extract the object and the background from the images. Trained ANN collects the input image pixels into two groups or clusters and produces the texture image segmentation and classification.

Wencang Zhao [28] proposed novel segmentation algorithm based on textural features and ANN to extract the regions of interest from the background. A set of micro-CT images are used. A pre-processing denoising filter is used forimage enhancement and noise removal from image, then feature extraction is carried out, after that, back propagation $\mathrm{NN}$ is set. Finally, the connection weights of network are modified, and the output is saved. The author compared their proposed algorithm with thresholding and region growing method. They claimed that their proposed approach performs well compared to the other methods according to the CPU time and the segmentation accuracy.

Lijun Zhang [29] proposed a new neural network based image segmentation system for color images. They combined the Wavelet Decomposition and Self Organizing Map (SOM) to propose a new method, i.e., SOM-NN. Voting among child pixels selected the parent 
pixel. After initialization, ANN found the segmentation result which satisfies all levels. Wavelet decomposition is performed to remove noise. Hence wavelet decomposition and SOM-NN are combined to perform segmentation. Results have shown that method has reduce noise and produce accurate segmentation.

\subsection{Edge Detection}

Edge detection [11] is an advanced technique in the field of image processing. Edges boundaries of a region are important features in the image, since there are often sharp transitions in the intensity at the region boundaries. Edge detection techniques are often combined with another segmentation technique where segmentation techniques can be applied to edges extracted from the edge detectors.

\subsection{Region Based Approaches}

\subsubsection{Region Growing}

Region growing segmentation technique is one of the simplest approaches to segment an image. Segmented region is formed from the neighboring pixels which have similar amplitude. This is achieved by finding a seed pixel in the region from which to grow that region. In the first stage of region growing segmentation process, the quantized pixels are grouped together in groups if they are four-connected are and of the same amplitude.

Region growing techniques start with one pixel (called the seed pixel) of a potential region then try to grow it by grouping adjacent pixels till the pixels being compared to be added are not similar. The basic idea of region growing approach can be summarized as follows:

- Random pixels (seed) are manually or automatically chosen.

- The 8-connected pixels and a predefined criterion are used to extract the connected pixels to the initial seed point.

- Repeat until all pixels are classified.

The first selected pixel (the seed) is chosen to be the first unlabeled pixel in the image or a number of seed pixels can be selected from the entire 
image. Adding pixel to a certain region needs some rules to be used to determine which pixels can be added to this region.

In region growing, two rules are needed to remove weak boundaries in the same region, control region growth and to check the homogeneity of the regions after each growth step.

At each iteration, and for each region, the growth mechanism checks if there are any unclassified pixels in the 8-neighborhood of the region borders. Then the homogeneity check rule is performed before locating a pixel x to a region $R_{i}(k)$, and the heuristic rule merges smaller regions into larger regions and prevent merging of adjacent regions of approximately the same size. The second rule removes weak common boundaries in the regions that remain after the first rule application.

This approach is used in remote sensing applications. Unfortunately, the region growing approach as many drawbacks such as, sensitivity to noise, also can cause extracted regions to have holes or discontinuities, also different sets of initial seed points cause different segmentation results, if the seed point was not chosen correctly, the segmentation fail. It is also time consuming.

\subsubsection{Region Splitting}

Region splitting starts with a number of seed points and regions are grown from these seeds by grouping the neighbor pixels with the same characteristics to its seed.

Region splitting is the opposite process of region growing. The image is assumed to be homogeneous, if not; the image is split into four regions or sub-images. The splitting procedure is iterative and will be repeated until the image is split into homogeneous segments or regions. One of the disadvantages of region splitting is that it may create adjacent segments that may be similar but will not be merged. 


\subsubsection{Region Splitting and Merging}

Region splitting and merging is an algorithm that consists of both region split and merge at each step. It is also related to region growing algorithm but it doesn't require choosing a seed point.

It is a top-down algorithm that assumes that the whole image is uniform and homogeneous. If this assumption is not true, it begins splitting the image into four parts (sub-images). If two neighboring regions $R_{i}, R_{i}$ are similar or uniform, the mean $M(R)$ and standard deviation ${ }^{s t d}(R)$ of these regions are computed. If $\left|M\left(R_{1}\right)-M\left(R_{2}\right)\right|<K * \min \left\{\operatorname{std}\left(R_{1}\right)\right.$, std $\left.\left(R_{2}\right)\right\}$, then they can be united $\left(P\left(R_{i} \cup R_{j}\right)=\right.$ true $)$. Split and merge algorithm is repeated until there is no possible split or merge.

Segmentation by splitting and merging techniques is based upon breaking the image into four parts (called quadrants), if the image is found to be originally non-uniform. Each of these quadrants is split into four parts again until the segments are found to be uniform. Adjacent pairs are merged if they are uniform enough. If neighboring squares are found to be uniform, then they are merged together into a single square which contains the four adjacent squares.

One advantage of splitting and merging approach is that it produces more compact regions than pure splitting algorithm. This approach is applicable in medical applications.

D. Barbosa [30] proposed a new technique for image segmentation that combines the region based and edge based data with spectral method using morphological watershed algorithms. Firstly, a preprocessing filter is used for noise removal with magnitude gradient, secondly, region merging is performed as a pre-segmentation, then region similarity graph is created and finally, Multi Class Normalized Cut method is used to perform the segmentation. Results show that this method performs well when compared to the spectral clustering method.

Tiancan Mei [31] claims that Markov random field (MRF) is suffered from lack of handling the large range of interaction. In order to overcome 
this drawback of MRF, they presented a new supervised image segmentation method, i.e., Region based Multi-scale segmentation method. Natural scene images are used as a dataset. By using region as a parameter in Multi-scale MRF model, their algorithms perform better than other techniques. It is observed from results that RSMAP improve the MSAP method used for image segmentation.

Gang Chen [32] found that fast extraction of object information from a given image is still a problem for real time image processing. They also found that region based methods are also time consuming and not give effective segmentation. They proposed a new region based method based on Least Square method in order to detect objects sharply. They used a weight matrix for region based method which also takes the local information into account and also the usage of Least Square method provides optimal and fast segmentation. Comparison of their method is conducted with Otsu method and Chan-Vese method using Lena image. Their method can extract the features more accurately than other methods.

Zhen H., Yewei L. [33] proposed new method for image segmentation which is based on region growing method and improved visual attention. Gabor filter and Guass-Laplace filters are used to extract the input image edges and the gray-level values. Then ANN methods are used to extract the region of interest. Experiments are conducted on natural scene images, and it is found that their algorithm not only segmented the image perfectly but also found the salience edges which others can't.

Fernando C. Monteiro [34] proposed new method for image segmentation which combines region based and edge based data with the aid of spectral method and watershed morphological algorithm. Firstly, bilateral filter is used to reduce the noise in the image. Secondly, pre-segmentation is performed using region merging, and region similarity is created then multi-class normalized cut method is used to perform graph based region grouping. The author compares their approach to mean shift, multi-scale graph based segmentation, and JSEG. They claimed that their proposed approach outperforms the other methods and better results are obtained. 


\subsection{Graph Based Methods}

Graph based methods partition image using matrices to yield a set of disconnected subgraphs. Let $G=(V, E)$ be an undirected graph with vertices $v_{i} \in \mathrm{V}$, the set of nodes or pixels to be segmented, and $\left(v_{i}, v_{i}\right) \in \mathrm{E}$ edge component connects neighbors $v_{i}$ and $v_{i}$. The edge is assigned a weight $w_{i j}$ based on the similarity between two elements or nodes $v_{i}, v_{i}$ [20], [21].

In segmentation of an image, the elements in this graph are pixels and the edge weightsare the measure of the similarity between the two pixels which are connected by this edge (e.g., the similarity in intensity, location, motion, color, or some other features). This means that edge between two pixels or nodes in the same region should have relatively low weights, and edges between pixels in different regions should have higher weights. Minimum cut is an example of graph based approaches. Graph based approaches are not popular in remote sensing applications.

Muhammad Rizwan Khokher [35] presented a new method of image segmentation using Fuzzy Rule based system and Graph Cuts. Authors have firstly segmented the gray scale, color, and texture images using Graph Cuts. Weights are assigned to the features of image using Fuzzy Rules. Their algorithm works by firstly extracting the features of image, calculate the constants using fuzzy rules, calculate the weighted average of constants to find the similarity matrix, partition the graph using Normalized Graph Cut method, and finally get the segmented image from partitioned graph. Berkley database is used to evaluate the algorithm. Simulation is performed in Matlab and C language. Results are evaluated on the basis of Mean, Standard Deviation, and PPV value.

\subsection{Watershed Segmentation}

The watershed algorithm [26, 34] uses the image gradient magnitude as a topographic surface. Pixels which have high gradient magnitude intensities (GMIs) values correspond to the lines of watershed that outline the boundaries of the region. Water placed on a certain pixel enclosed by a common watershed line flows downhill to a common Local Intensity 
Minimum (LIM). Pixels draining to the same minimum form a catch basin, which represents a segment.

\subsection{Deformable Models}

Segmentation techniques based on deformable model can outperform many of the traditional image segmentation techniques drawbacks. These models take into account the boundary of object of interest and make use of a priori knowledge of object shape to improve the segmentation process. The deformable models can handle noise and other artifacts in object edges and boundaries.

Deformable models include two main approaches, the parametric approaches and the level set methods. The parametric approaches, also referred to as snakes, are not only compact, but also they can overcome both the noise in the image and boundary irregularities and gaps as they force the extracted boundaries to be smooth. In contrast, level sets are prepared to process the changes in the image. However, unlike the parametric approaches, they are not robust to boundary gaps and irregularities and they also complain different other problems.

\subsubsection{Parametric Model(Snakes)}

The energy function for snakes:

$$
v(s)=((\mathrm{x}(\mathrm{s}), \mathrm{y}(\mathrm{s}))
$$

is given by:

$$
E_{\text {snake }}=\int_{0}^{1} E_{\text {int }}(v(s))+E_{\text {image }}(v(s))+E_{\text {con }}(v(s)) d s
$$

Where $E_{\text {int }}$ is the internal energy derived from the contour model, used to control the shape and depend on the curvature of the snake. It can control how sharply the curve can bend. The external energy derived from the image features is usefulin extracting the boundary of the object of interest.

$E_{\text {image }}$ is the image energy that depends on the type of feature towards which the snake will be attracted. If $E_{\text {image is inversely proportional to the }}$ 
magnitude of the image gradient, the snake will be attracted to the image edges. $E_{\text {con }}$ provides user specific control.

Given the initial curve, an iterative procedure is used to derive the snake towards the state of minimum energy calculated based on the gradient decent and the initial position of the curve will determine the final location of the contour.

Snakes are extensively used in segmenting medical images and their advantages are that they are robust to noise and suitable for smooth images with simple background, however, not without limitations.

One of the snakes' limitations is the problem of separating multiple objects that requires initialization of multiple snakes that will increase complexity of the segmentation process. To solve this problem, initial seed contours all over the image are selected. These seed contours are initially small and are gradually grow. The contours energy functional is normalized by contour length to avoid bias towards larger contours.

It is possible for the snake to stuck at one such minimum and miss the global minimum, since the energy function usually has many local minima. This can be solved using Balloon snakes.

\section{- Balloon Snakes}

In Balloon snakes an additional force, called pressure force, is added to the traditional active contour, given by:

$$
f_{\text {ballon }}=\overrightarrow{f_{\text {unt }}}+\overrightarrow{f_{\text {ext }}}+\overrightarrow{f_{\text {pressure }}}
$$

Like the balloon, the curve expands and retracts according to the initial contour position. It is used to overcome the local minima because the additional pressure force causes the Balloon to pass the local minima and stop at high contrast edges or global minimum.

Limitations of Balloons are that they fail to capture contours with discontinuities and fail to move along objects with concaved regions as it uses the same external force of the traditional snakes. 


\section{- GVF Snake}

The Gradient Vector Flow (GVF) snake model solves two problems; poor performance for concave boundaries and poor performance when snake is initialized far from the optimum position.

GVF snake is created from vector field $V_{G V F}=\left[u_{G V F}(x, y), v_{G V F}\right]$ which is calculated by the fusion of the gradient vectors which are defined as the edge map of binary image or gray levels. This vector field replaces the potential external force, which define the equilibrium condition of the equation $V_{G V F}=\left[u_{G V F}(x, y), v_{G V F}\right]$

A traditional snake should be initiated near the boundary; however, it still cannot reach to boundary cavities. But the main advantages of the GVF snake is that it can start far away from the desired boundary and will converge to boundary cavities and concavities, also the GVF snake can be started across the boundaries, which is impossible for traditional and balloon snakes. Not only can GVF snakes handle gray-scale images but also it is suitable to handle binary images. One disadvantage of GVF is the minimization of the cost function problem which is non-trivial and imposes certain smoothness constrains on the solution.

Ping Jiang, Quansheng Dou and Xiaoying $\mathrm{Hu}$ [36] proposed a parallel realization of the active contour model on boundary extraction in biomedical images such as blood vessel tracing or optic disc extraction in retinal images. The initially drawn contour is split into two or more independently controlled sub-contours, and each sub-contour converges independently in parallel. The contour is split into two, four or more subcontours depending on the object geometry and its complexity. The subcontours are assigned to different threads that can run in parallel, and then these sub-contours can converge to the boundaries of the objects. Finally, the converged sub-contours are joined together to obtain the whole object contour. Combining the individual sub-contours results in obtaining the final true contour. The contour convergence in the parallel realization version is more efficient when compared to the serial realization. The author stated that their approach is more efficient when compared to the other parallel algorithms since the method requires minimal coordination between parallel threads. 


\section{- Radial Active Contour Method (pSnakes)}

The pSnake radial active contours methods make use of polar coordinates $(r, \theta)$ where ${ }^{r}$ is the radius and $\theta$ is the angle representing the image pixels in the ultrasonic mode. The reference axis origin is located at the point of divergence from the ultrasonic beam, i.e., at the point where the ultrasound probe is located.

A.A. Alexandria, P. C. Cortez, J. A. Bessa, J. H. S. Felix, J. S. Abreu and V. H. Albuquerque [37] proposed a novel approach which proposes a new radial active contour technique called pSnake making external energy to be 1D Hilbert transform which is the main contribution of this work. The pSnake approach is built on the theory that the beams in ultrasonic mode diverges from a single point which enable the use of polar coordinates in image segmentation process. The active contour control points or nodes, called twin nodes, are acquired in pairs. The internal and external energies are redefined. The pSnake method is not affected by variations in image contrast and noise so, it is more suitable for ultrasound images. The pSnake method is compared to a variety of methods and results shoes that it is fast, very robust and the most accurate for all situations even in the presence of speckle noise.

\subsubsection{Level Set Method}

Level Sets are considered very important class of sophisticated image segmentation methods that are based upon Partial Differential Equations (PDE) that depends on progressive evaluation of the difference between adjacent neighbor pixels to determine the desired object edges or boundaries. In addition, three dimensional surfaces can be determined as well because the algorithm operates in three dimensions with almost no change.

Like the active contour models, this algorithm has a speed function that is calculated from the image gradient. Level set methods are used intensively for segmenting medical image where it can manage the cavities and concavities, splitting or merging.

John A. Bogovic, Jerry L. Prince and Pierre-Louis Bazin[38] proposed a Multiple-object Geometric Deformable Model (MGDM) for image 
segmentation. The authors present a multiple object segmentation method using a novel and efficient object representation for both two and three dimensions which guarantees no overlaps or gaps and can preserve the topology of all objects. MGDM is compared to the multiphase approach and the $\mathrm{N}$ coupled level sets of Brox and Weickert. Numerical results demonstrate the accuracy and stability of the method. The author claimed that MGDM method is well-suited to many new challenging segmentation problems since it accurately and efficiently can outline objects. MGDM can be applied to cell segmentation and tracking retinal layer segmentation from Optical Coherence Tomography (OCT) images and segmentation of ultra-high resolution brain image.

Zhen Yang, John A. Bogovic, Aaron Carass, Mao Ye, Peter C. Searson and Jerry L. Prince [39] proposed a fully automatic method for segmenting cells in fluorescence images of confluent cell monolayers. Firstly, initial seeds are found and extracted from the detected cell nuclei for segmentation. The order-statistic filtering and a principal curvature based image operator enhance the cell junction. The MGDM is used in the final segmentation that proposes a robust and accurate segmentation and guarantees no gaps and overlaps between neighbor cells.

Paresh Chandra Barman et.al [40] proposed a new image segmentation approach using medical diagnosis system. The system uses a new variational level set algorithm with no re-initialization that can be executed by a simple finite difference scheme. Thresholding and erosion algorithms are used to remove noise of the image. The system results in a quick detection of the initial curve and the interior contours (like tumors).

Jinsheng Xiao [41] proposed a new non-linear discontinue partial differential equation (PDE) that models the level set method of gray images. A discrete method is also proposed to find numerical solution and to implement the filter. Non-Linear discontinue PDE formula is applied on image of cameramen using MATLAB. Results have shown that image edges and boundaries are remained blurred and can be shifted by using Close operator. More information can be saved by using the proposed scheme.

There are also a set of researches that discussed the existing segmentation algorithms, some of these studies are submitted. 
Kabade Mankarnika Manohar and A. S. Patil [42] covered a review on various segmentation techniques such as thresholding, region based segmentation, edge based segmentation, and clustering approach. Not all segmentation techniques are useful for all types of images, i.e. it is found that there is no perfect image segmentation technique because the result of image segmentation depends on many factors such as intensity texture, pixel color, similarity of images, image content and the domain problem. So it is not possible to use a single method for all types of images nor all methods can perform well for all types of images.

Sonam Mandiratta, PoojaBatra Nagpal and Sarika Chaudhary [43] discussed the various methods of image segmentation process such as Thresholding based, Clustering based, Fuzzy based, Edge based, ANN based, Region based and Watershed based. A summary of image segmentation methods is presented in this review paper. The author concluded that image segmentation is an important aspect and it has a lot of work to be done in the future.

Aman Mittal [44] presented a review the different techniques of image segmentation including Thresholding based, Clustering based, Edge based and Region based and also the diagrammatic difference between the all these four technique. By having a brief insight over all the segmentation technique in this research, it may be concluded that the choice of the segmentation technique depends upon the requirements of the application and there is no general technique available for all image segmentation applications, the technique is chosen according to the application that we are processing.

Arti Taneja, Priya Ranjan and Amit Ujjlayan [45] proposed a study of various segmentation techniques like edge, threshold, region, clustering and neural network involved in the effective image analysis. The efficiency of the segmentation process improved with the help of several algorithms, namely, active contour, level set, Fuzzy clustering and Kmeans clustering. This research analyses the performance of algorithms for image segmentation. Segmentation based on active contour models conveyed that refinement of boundary was an important process in segmentation. Grouping of pixels of an image led the development of fuzzy based and K- means clustering process. The kernel metric approaches in addition to clustering eliminated the need of prediction of the number of clusters. K-means processes applied in various real world 
images such as brain MRI images, tumor segmentation for maximum accuracy. The research also proposed a multilevel set segmentation based on the combination of intensity and texture with level set functions. It is find out that intensity and texture based methods based on level set function efficiently segment the image. The quality of the image with the presence of noise analyzed and improved on texture based methods and the effective refinement of boundary on Optical Disk (OD) in retinal images performed by the level set methods.

Amanpreetkaur and Navjotkaur [46] proposed a survey on image segmentation improving methods using different techniques. This paper presents a brief outline on some of the most commonly used segmentation techniques like Thresholding based, Fuzzy based, Edge based, ANN based and Region based. The author focused on the top five techniques used for image segmentation and explain the details about each technique and how the segmentation is achieved. Not all segmentation techniques are useful for all types of images, there is no single image segmentation technique that performs well with all types of images but each technique is good for particular type of images.

Priyanka Shivhare and Vinay Gupta [47] proposed a discussion on image segmentation and its different techniques that are used in various fields such as biomedical field, computer vision and image processing. The review is aimed at providing an overview of current image segmentation techniques; therefore, analysis and comprehensive assessment of these image segmentation techniques are done. Based on this, it is concluded that image segmentation has a promising future and a lot of research work is required for developing a common and consistent segmentation technique which can be applied universally.

Pooja Rani and Rakesh Verma [48] reviewed various ultrasound image segmentation techniques like Edge Based Approaches, Region Based Approaches, Otsu's Algorithm, Genetic Algorithm, B-Spline Method, Active Contour Method, Fast-Marching Method and Watershed Segmentation.

Muhammad Waseem Khan [49] presents a literature review of basic image segmentation techniques from last few years. The review discussed all basic image segmentation techniques currently being used by the researchers and industry 
M. J. Kumar, GVS Raj Kumar and R. Vijay Kumar Reddy [50] proposed a review study for different image segmentation techniques such as thresholding, edge based segmentation, region based segmentation, Neutral Network and also covered finding threshold value for it and the advantages and disadvantages for each technique

R. Kandwal, A. Kumar and S. Bhargava [51] proposed a survey on the current image segmentation techniques such as Segmentation based on edge detection, Threshold Method, Region based Segmentation Methods, Clustering Techniques, Neural Network-based segmentation and Model Based Segmentation including the explanation for each technique. The authors concluded that there is no single algorithm that is applicable on all types of images and nature of problem so, image segmentation still remains a big pending problem in the areas of image processing.

Anamika Ahirwar [52] investigates the use of different image segmentation techniques that are used to detect and classify the image regions. The authors also discuss some segmentation techniques classified by researchers. Segmentation and characterization of Brain MR image regions using SOM and Neuro fuzzy techniques, such as Self Organizing Map (SOM) and Neuro Fuzzy scheme to automatically extract White Matter(WM), Gray Matter(GM), Cerebrospinal Fluid (CSF) and tumor region of brain MRI image tested on three normal and three abnormal brain MRI images. Using some statistical tests like precision, sensitivity, accuracy, specificity, negative predictive value, positive predictive value, false negative rate, false positive rate, likelihood ratio negative, likelihood ratio positive and prevalence of disease. Finally, the effectiveness of each method is investigated.

Cristina S. and Ştefan H. [53] presented a paper to review the most important image segmentation methods including classical image segmentation methods (thresholding, regions-based, and edges-based), pattern recognition-based, deformable models, wavelets-based methods, and atlas-based techniques and discuss the principle and the mathematical model for each method. The research concluded that thresholding is one of the simplest segmentation techniques. The disadvantage of thresholding methods is that they can be applied to a single-band image, such as a grayscale image or a single band of a multi-band image. Region based methods have shown to be very useful and efficient segmentation techniques in image processing. However, they have over-segmentation 
tendency, require manual initialization and are sensitive to noise. Clustering technique can be used for multi-band images, but the number of groups must be established first. Classification-based algorithm requires a training phase. Deformable models are less sensitive to noise than the other techniques, which make them suitable for complex medical image segmentation problems. Atlas-based methods use prior knowledge in order to perform segmentation, but they are time-consuming. Generally, thresholding, edge-based, region based, and classification-based algorithms can solve simple medical image segmentation problems. For complex medical images, which cannot be handled robustly by general segmentation methods, deformable models and atlas-based segmentation methods are the most appropriate.

Ajala F. A. et.al [54] discussed a set of methods used to segment medical image such as Thresholding, Clustering, Classifier, Region Growing, Markov Random Model, Deformable Model, etc. This survey has mainly focused on clustering methods (k-means and fuzzy c-means clustering algorithms). They merge the two algorithms together to create another technique called fuzzy K-C means clustering algorithm. The algorithms have been applied on MRI human brain images. This technique results in a better performance based on CPU time computation.

Krishna Kant Singh and Akansha Singh [55] proposed a survey on the various image segmentation techniques that are available for text, color images and gray scale images. This study proposes different types of image segmentation techniques such as Pixel-Based Segmentation, EdgeBased Segmentation, Region-based, Model-Based Segmentation, etc. The survey stated that the suitable segmentation algorithm is determined based on the type of input image and the application. In this study, the author has discussed and proposed a few segmentation algorithms which also take into consideration the type of input image like color, gray scale and text.

Table (1) displays a comparison between the various medical image segmentation techniques showing their advantages and limitations and the specific application adopted for each. 
Table1: Comparison of medical image segmentation techniques.

\begin{tabular}{|c|c|c|c|}
\hline Technique & Advantages & Disadvantages & $\begin{array}{c}\text { Type of } \\
\text { Application }\end{array}$ \\
\hline $\begin{array}{l}\text { Thresh- } \\
\text { olding }\end{array}$ & $\begin{array}{l}\text { - Simple } \\
\text { method. } \\
\text { - Easy to } \\
\text { implement. } \\
\text { - Used in real- } \\
\text { time } \\
\text { applications. }\end{array}$ & $\begin{array}{l}\text { - Sensitive to noise. } \\
\text { - Choosing the accurate } \\
\text { threshold value is } \\
\text { difficult task. } \\
\text { - Doesn't work well } \\
\text { when dealing with flat } \\
\text { images with smooth } \\
\text { peaks and valleys. } \\
\text { - Not suitable to handle } \\
\text { images with high } \\
\text { variability of features. }\end{array}$ & $\begin{array}{ll}\text { - Medical } \\
\text { image (gray- } \\
\text { scale images) } \\
\text { segmentation. } \\
\text { - Pharmaceutic } \\
\text { al industry. } \\
\text { - Finger vein } \\
\text { image } \\
\text { segmentation. }\end{array}$ \\
\hline Clustering & $\begin{array}{l}\text { - Remove the } \\
\text { noisy parts. } \\
\text { - Easy to } \\
\text { complement } \\
\text { - } \begin{array}{l}\text { Fast for } \\
\text { small no. of } \\
\text { clusters. }\end{array}\end{array}$ & $\begin{array}{l}\text { - Difficult to estimate } \\
\text { the appropriate no. of } \\
\text { clusters and the initial } \\
\text { centers especially at } \\
\text { high variability of } \\
\text { features } \\
\text { - It is expensive. } \\
\text { - Time consuming } \\
\text { - Not suitable for large } \\
\text { data base. }\end{array}$ & $\begin{array}{l}\text { - Medical } \\
\text { imagery. } \\
\text { - MRI image } \\
\text { segmentation. }\end{array}$ \\
\hline $\begin{array}{l}\text { Graph } \\
\text { Based }\end{array}$ & $\begin{array}{l}\text { Efficient } \\
\text { method and } \\
\text { accepted } \\
\text { world-wide. } \\
\text { Can achieve } \\
\text { globally } \\
\text { optimal } \\
\text { result for } \\
\text { the energy } \\
\text { function. } \\
\text { - Effective to } \\
\text { the natural } \\
\text { image } \\
\text { without any } \\
\text { pre-known } \\
\text { information. }\end{array}$ & $\begin{array}{l}\text { - Memory and CPU time } \\
\text { limitations. } \\
\text { - Hard to segment thin } \\
\text { elongated structures. }\end{array}$ & $\begin{array}{ll}\text { - } & \text { gray scale } \\
\text { images } \\
\text { - } & \text { color images } \\
\text { - } & \text { Texture } \\
& \text { images } \\
& \text { segmentation. }\end{array}$ \\
\hline
\end{tabular}




\begin{tabular}{|c|c|c|c|}
\hline Technique & Advantages & Disadvantages & $\begin{array}{c}\text { Type of } \\
\text { Application }\end{array}$ \\
\hline Edge Based & $\begin{array}{l}\text { Works well } \\
\text { on images } \\
\text { with best } \\
\text { contrast } \\
\text { between } \\
\text { objects and } \\
\text { background. }\end{array}$ & $\begin{array}{l}\text { - Does not work well on } \\
\text { images and edges with } \\
\text { low contrast }\end{array}$ & $\begin{array}{l}\text { - Medical } \\
\text { image } \\
\text { segmentation. }\end{array}$ \\
\hline $\begin{array}{c}\text { NN } \\
\text { Techniques }\end{array}$ & $\begin{array}{l}\text { Does not } \\
\text { dependent } \\
\text { on the } \\
\text { probability } \\
\text { density } \\
\text { distribution } \\
\text { function. } \\
\text { No } \\
\text { complicated } \\
\text { programs. }\end{array}$ & $\begin{array}{l}\text { - } \begin{array}{l}\text { Requires three sets of } \\
\text { data. }\end{array} \\
\text { - } \quad \text { Long training time. } \\
\text { Have difficulties when } \\
\text { segmenting image } \\
\text { affected by changes in } \\
\text { the tissue } \\
\text { characteristics. } \\
\text { Not effective for real } \\
\text { time applications. }\end{array}$ & 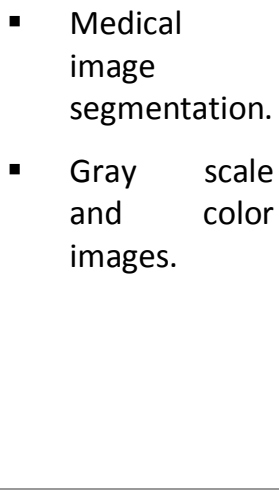 \\
\hline $\begin{array}{l}\text { Water Shed } \\
\text { Techniques }\end{array}$ & $\begin{array}{l}\text { - Fast } \\
\text { computatio } \\
\text { n speed. }\end{array}$ & - Over segmentation. & $\begin{array}{l}\text { - Gray scale } \\
\text { image } \\
\text { segmentation }\end{array}$ \\
\hline $\begin{array}{l}\text { Level set } \\
\text { Method }\end{array}$ & $\begin{array}{l}\text { - Can manage } \\
\text { the cavities } \\
\text { and } \\
\text { concavities, } \\
\text { splitting or } \\
\text { merging. } \\
\text { - Three } \\
\text { dimensional } \\
\text { surfaces can } \\
\text { be } \\
\text { determined. } \\
\text { - No gaps or } \\
\text { overlaps }\end{array}$ & - $\quad$ Long CPU time & $\begin{array}{l}\text { - Gray scale } \\
\text { image } \\
\text { segmentation. }\end{array}$ \\
\hline
\end{tabular}

\section{Freely and Commercial Software Packages for Medical Image Segmentation}


There are many software packages and libraries for medical image segmentation [56]. In this section the different software packages and their requirements are stated.

Table (1) describes the various software packages for medical image segmentation and their requirements.

Table (2) The different commercial software tools for medical image segmentation and their requirements.

\begin{tabular}{|c|c|c|c|c|c|c|}
\hline Software & Description & $\begin{array}{c}\text { Software } \\
\text { type }\end{array}$ & Interface & $\begin{array}{c}\text { Restrict } \\
\text { ions to } \\
\text { use }\end{array}$ & $\begin{array}{c}\text { Operating } \\
\text { system }\end{array}$ & $\begin{array}{c}\text { Program } \\
\text { ming } \\
\text { languages }\end{array}$ \\
\hline ITK-SNAP & 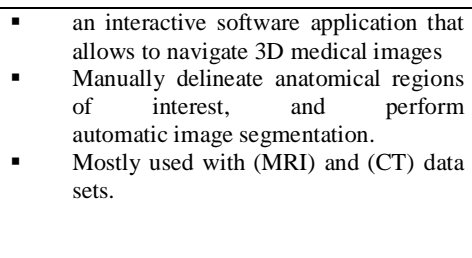 & Toolkit & $\begin{array}{l}\text { Graphical } \\
\text { user } \\
\text { interface, } \\
\text { Command } \\
\text { line } \\
\text { interface. }\end{array}$ & None & $\begin{array}{l}\text { Mac OS, } \\
\text { Linux, and } \\
\text { Windows }\end{array}$ & $\mathrm{C}++$ \\
\hline VTK & $\begin{array}{l}\text { An open-source, freely available } \\
\text { software system for 3D computer } \\
\text { graphics, image processing and } \\
\text { visualization. }\end{array}$ & Toolkit & $\begin{array}{l}\text { Graphical } \\
\text { user } \\
\text { interface }\end{array}$ & None & $\begin{array}{l}\text { Windows } \\
\text { Vista, Mac } \\
\text { OS X, } \\
\text { Linux }\end{array}$ & $\begin{array}{l}\text { C++, Java, } \\
\text { Python }\end{array}$ \\
\hline ITK & $\begin{array}{l}\text { An open-source, cross-platform system } \\
\text { that provides developers. } \\
\text { Developed through extreme } \\
\text { programming methodologies. } \\
\text { Employs leading-edge algorithms for } \\
\text { registering and segmenting } \\
\text { multidimensional data. }\end{array}$ & Toolkit & $\begin{array}{l}\text { Graphical } \\
\text { user } \\
\text { interface }\end{array}$ & None & $\begin{array}{l}\text { Windows, } \\
\text { Linux, } \\
\text { Mac OS X }\end{array}$ & $\mathrm{C}++$, Java \\
\hline SPM & $\begin{array}{l}\text { The SPM software package has been } \\
\text { designed for the analysis of brain } \\
\text { imaging data sequences. The sequences } \\
\text { can be a series of images from different } \\
\text { cohorts, or time-series from the same } \\
\text { subject. The current release is designed } \\
\text { for the analysis of fMRI, PET, SPECT, } \\
\text { EEG and MEG. }\end{array}$ & Package & $\begin{array}{l}\text { graphical } \\
\text { user- } \\
\text { interface }\end{array}$ & None & Mac OS & MATLAB \\
\hline MIA & $\begin{array}{l}\text { A general-purpose image processing } \\
\text { toolbox used in 2D and 3D gray scale } \\
\text { medical image analysis. } \\
\text { Uses a test-driven development to } \\
\text { ensure reliability of the implementation. }\end{array}$ & Plug-in & $\begin{array}{l}\text { Command } \\
\text { line } \\
\text { interface }\end{array}$ & None & $\begin{array}{l}\text { Linux, } \\
\text { Windows } \\
\text { Mac OS }\end{array}$ & $\mathrm{C}++$ \\
\hline NiftySeg & $\begin{array}{l}\text { - Performs EM based segmentation or } \\
\text { analyses images. } \\
\text { Contains a package of label fusion } \\
\text { algorithms (MV, STAPLE, and SBA) } \\
\text { with different types of ranking } \\
\text { strategies. }\end{array}$ & Package & $\begin{array}{l}\text { Command } \\
\text { line } \\
\text { interface }\end{array}$ & None & $\begin{array}{l}\text { Windows, } \\
\text { Linux, } \\
\text { Mac OS X }\end{array}$ & $\begin{array}{l}\text { MATLAB, } \\
\mathrm{C}++\end{array}$ \\
\hline GIMIAS & $\begin{array}{l}\text { GIMIAS is a workflow-oriented } \\
\text { environment for solving advanced } \\
\text { biomedical image computing and } \\
\text { individualized simulation problems, } \\
\text { which is extensible through the } \\
\text { development of problem-specific plug- } \\
\text { ins. } \\
\text { Provides an open source framework for } \\
\text { efficient development of research and }\end{array}$ & $\begin{array}{l}\text { Plug-in } \\
\text { framework }\end{array}$ & $\begin{array}{l}\text { graphical } \\
\text { user- } \\
\text { interface }\end{array}$ & None & $\begin{array}{l}\text { Windows, } \\
\text { Linux }\end{array}$ & $\mathrm{C}++$ \\
\hline
\end{tabular}




\begin{tabular}{|c|c|c|c|c|c|c|}
\hline & $\begin{array}{l}\text { clinical software prototypes integrating } \\
\text { contributions from the Physiome } \\
\text { community while allowing business- } \\
\text { friendly technology transfer and } \\
\text { commercial product development. } \\
\text { Provides manual and automatic } \\
\text { segmentation, visualization, mesh } \\
\text { editing and electro mechanical and CFD } \\
\text { simulation among others. }\end{array}$ & & & & & \\
\hline Software & Description & $\begin{array}{c}\text { Software } \\
\text { type }\end{array}$ & Interface & $\begin{array}{c}\text { Restrict } \\
\text { ions to } \\
\text { use }\end{array}$ & $\begin{array}{c}\text { Operating } \\
\text { system }\end{array}$ & $\begin{array}{c}\text { Program } \\
\text { ming } \\
\text { languages }\end{array}$ \\
\hline 3D Slicer & $\begin{array}{l}\text { A free, open source software package } \\
\text { for visualization and image analysis. }\end{array}$ & Package & $\begin{array}{r}\text { graphical } \\
\text { user- } \\
\text { interface }\end{array}$ & None & $\begin{array}{l}\text { Windows, } \\
\text { Linux } \\
\text { Mac Os X }\end{array}$ & $\begin{array}{r}\text { C++, } \\
\text { Python }\end{array}$ \\
\hline $\begin{array}{l}\text { Amira 3D } \\
\text { Software for } \\
\text { Life Sciences }\end{array}$ & $\begin{array}{l}\text { A powerful, multifaceted 3D software } \\
\text { platform for visualizing, manipulating, } \\
\text { and understanding data from computed } \\
\text { tomography, microscopy, MRI, and } \\
\text { many other imaging modalities. } \\
\text { Fast and flexible 3D imaging } \\
\text { workflows for molecular and cellular } \\
\text { biology to neuroscience and } \\
\text { bioengineering. }\end{array}$ & Package & $\begin{array}{r}\text { Graphical } \\
\text { user } \\
\text { interface }\end{array}$ & $\begin{array}{r}\text { License } \\
\text { purchas } \\
\text { e } \\
\text { required }\end{array}$ & $\begin{array}{r}\text { Unix/Linu } \\
\text { x, Mac } \\
\text { OS, } \\
\text { Windows }\end{array}$ & $\mathrm{C}++$ \\
\hline $\begin{array}{c}\text { Cell } \\
\text { Cognition }\end{array}$ & $\begin{array}{l}\text { A computational framework to annotate } \\
\text { complex cellular dynamics. } \\
\text { Combines state-of-the-art classification } \\
\text { with hidden Markov modeling for } \\
\text { annotation of the progression through } \\
\text { morphologically distinct biological } \\
\text { states. } \\
\text { Open source software, enabling live- } \\
\text { cell imaging-based screening with } \\
\text { assays that directly score cellular } \\
\text { dynamics. }\end{array}$ & $\begin{array}{r}\text { Framewor } \\
\mathrm{k}\end{array}$ & $\begin{array}{r}\text { Command } \\
\text { line } \\
\text { interface }\end{array}$ & None & $\begin{array}{r}\text { Unix/Linu } \\
\text { x, Mac } \\
\text { OS, } \\
\text { Windows }\end{array}$ & Python \\
\hline $\begin{array}{c}\text { Cell } \\
\text { Explorer }\end{array}$ & $\begin{array}{l}\text { A package of computer programs to } \\
\text { process and analyze 3D confocal image } \\
\text { stacks of the model animal C. elegans, } \\
\text { Can be applied to several other model } \\
\text { systems such as fruit fly embryo/larvae } \\
\text { as well. } \\
\text { Some other applications include 3D } \\
\text { cell/nuclei segmentation, quantification, } \\
\text { gene expression analysis, automatic cell } \\
\text { naming/annotation, cell targeting, etc. }\end{array}$ & Package & $\begin{array}{r}\text { Command } \\
\text { line } \\
\text { interface }\end{array}$ & None & $\begin{array}{r}\text { Unix/Linu } \\
\text { x, Mac } \\
\text { OS, } \\
\text { Windows }\end{array}$ & MATLAB \\
\hline $\begin{array}{c}\text { Morpho } \\
\text { Lib J }\end{array}$ & 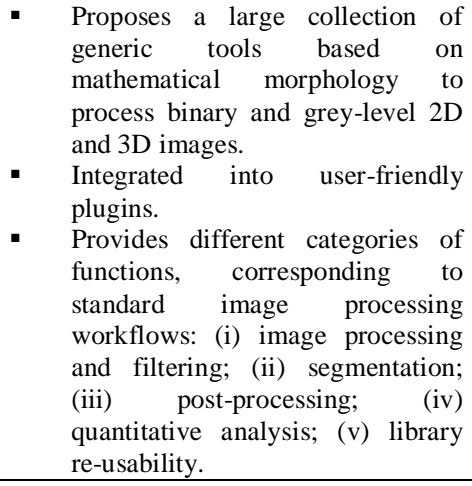 & Plug-in & $\begin{array}{r}\text { Graphical } \\
\text { user } \\
\text { interface }\end{array}$ & None & $\begin{array}{r}\text { Unix/Lin } \\
\text { ux, Mac } \\
\text { OS, } \\
\text { Windows }\end{array}$ & Java \\
\hline LEVER & $\begin{array}{l}\text { - } \quad \text { s software tool for lineage editing } \\
\text { and validation. } \\
\text { - } \quad \text { Segments, tracks and lineages time }\end{array}$ & Package & $\begin{array}{r}\text { Comman } \\
\text { d line }\end{array}$ & None & $\begin{array}{l}\text { Unix/Lin } \\
\text { ux, Mac }\end{array}$ & $\begin{array}{r}\text { MATLA } \\
\text { B }\end{array}$ \\
\hline
\end{tabular}




\begin{tabular}{|l|l|l|l|l|r|r|}
\hline & \multicolumn{1}{|l|}{$\begin{array}{l}\text { lapse microscopy image sequences } \\
\text { of live stem cells. } \\
\text { Works in 2-D and 3-D and with } \\
\text { phase and fluorescence images. }\end{array}$} & OSterface & & Windows & \\
\hline
\end{tabular}

\section{Some Novel Trends for Medical Image Segmentation}

It can be seen that there are several apparent trends for image segmentation study. It is important to pay attention to the existing methods while proposing new methods and concepts and the problems existing now will find acceptable solutions. In this part we focus on the novel trends for medical image segmentation which may include the following points:

- The original algorithms need continuous improvements which means strengthen the strong points and weaken the weak points.

- Hybrid approaches that means combining several approaches can also be used.

- The major problem with the current level set approach for segmentation is the long execution time, especially for volumetric images. This is typical for level set methods and can be improved somewhat with optimized implementations.

- Investigating alternative, probably simpler, methods for segmentation based on the deformable model.

- Choosing the weights and parameters of the snake model is an important and often tedious task.

- Application of the snake to practical problems to customize the snake models to practical biomedical problems. This will require several enhancements ranging from the choice of the image energy and type of shape constraint.

- Additional or enhanced energy terms and more human knowledge should be incorporated into the snakes design to further automate and improve segmentation.

- 3-D active contour model by extending the model-based consistent segmentation using a steerable feature space to 3-D. The parametric representation of general 3-D surfaces is difficult, unless the shapes assume simple forms like a tube; in this case the surface can be represented as using a spline 
model. Another promising approach may be to represent the shape using spherical harmonics.

- Account for the exact derivatives of the unified image energy: At the moment, the parameters of the probability distribution functions are assumed to be constant. However, in practice these parameters are estimated from the images depending of the current position of the contour. Thus these parameters are dependent on the curve coefficients. We would like to compute the exact directional derivatives in the optimization scheme and study the improvement.

- 3-D steerable feature detectors to generate a steerable feature space from the volume data and use it to detect specific features like lines, surfaces, edges, blobs etc.

- Multi-scale detection of image features and denoising.

\section{Conclusion}

This paper presents a review for the most important image segmentation techniques. It is concluded that each technique has its advantages and limitations and the suitable segmentation algorithm is determined based on the type of input image and the application. Based on this, it is concluded that medical image segmentation has a promising future and a lot of research work is required for developing a common segmentation technique which can be applied universally, since there is no single algorithm which can be applicable for all types of images.

It is found that Thresholding is one of the oldest and the simplest segmentation techniques. They have been a popular for many years because of the advantages of simplicity and ease of implementation. But it doesn't work well when dealing with flat images with smooth peaks and valleys. Region based methods have been very useful and efficient segmentation techniques but they are sensitive to noise, also can cause extracted regions to have holes or discontinuities. If the seed point was not chosen correctly, the segmentation fails. It is also time consuming. Clustering technique can be used for multi-band images, but the number of clusters must be determined at the beginning of the process. ANN requires a training phase. Deformable models are less sensitive to noise than the other techniques presented in this study, which make them suitable for complicated segmentation problems of medical image. Image segmentation using deformable models shows that improvement of boundaries was an important process in segmentation. The deformable model is the most robust and accurate technique for all situations even in 
the presence of speckle noise. The paper ends with highlighting on some novel trends for image segmentation.

\section{References}

[1] Seyed Masoud Nosrati, "Prior Knowledge for Targeted Object Segmentation in Medical Images", Ph. D thesis, School of Computing Science Faculty of Applied Sciences, 2015.

[2] SEDA ÇAMALAN, "Analysis of Filtering and Quantization Preprocessing Steps in Image Segmentation", Ms. C Thesis, Department of Computer Engineering, the Graduate School of Natural and Applied Science, July 2013.

[3] Bo XIANG, "Knowledge-Based Image Segmentation Using Sparse Shape Priors and High-Order MRFs", Ph. D thesis, Ecole Centrale de Paris, CVN laboratory, 2013.

[4] Gunnar Läthén,"Segmentation Methods for Medical Image Analysis Blood vessels, multi-scale filtering and level set methods", Ph. D thesis, Department of Science and Technology Linköping University, Sweden Norrköping, April 2010.

[5] Mohammad Shajib Khadem, "MRI Brain Image Segmentation Using Graph Cuts", Ms. C Thesis, Department of Signals and Systems, Chalmers University of Technology Göteborg, Sweden, 2010.

[6] Mahshid Roumi, "Implementing Texture Feature Extraction Algorithms on FPGA", Ms. C Thesis, Department of Electrical Engineering, Faculty of Electrical Engineering, Mathematics and Computer Science, Delft University of Technology, 2009.

[7] Francisco J. Estrada, "Advances in Computational Image Segmentation and Perceptual Grouping", Ph. D thesis, Department of Computer Science, University of Toronto, 2005.

[8] http://www.slideshare.net/AboulEllaHassanien/medical-image-analysis-27297012 (Access date, August 2016).

[9] http://www.powershow.com/view1/105a82ZDc1Z/Medical_Image_Segmentation_ powerpoitppt_presentation (Access date, August 2016).

[10] Li Haitao and Li Shengpu, "An Algorithm and Implementation for Image Segmentation", International Journal of Signal Processing, Image Processing and Pattern Recognition Vol.9, No. 3, pp. 125-132, 2016.

[11] M. J. Islam, S. Basalamah, M. Ahmadi, and M. A. S. Ahmed, "Capsule Image Segmentation in Pharmaceutical Applications Using Edge-Based Techniques", IEEE International Conference on Electro/Information Technology (EIT), pp. 1-5, 2011.

[12] W. Kaihua and B. Tao, "Optimal Threshold Image Segmentation Method Based on Genetic Algorithm in Wheel Set Online Measurement," in Proc. Third International Conference on Measuring Technology and Mechatronics Automation (ICMTMA), pp. 799-802, 2011.

[13] Salem Saleh Al-amri, N.V. Kalyankar and Khamitkar S. D., "Image Segmentation by Using Threshold Techniques", Journal of Computing, Vol. 2, No. 5, May 2010.

[14] W. Cui and Y. Zhang, "Graph Based Multispectral High Resolution Image Segmentation", in Proc. International Conference on Multimedia Technology (ICMT), pp. 1-5, 2010. 
[15] F. Zhang, S. Guo, and X. Qian, "Segmentation for Finger Vein Image Based on PDEs Denoising", in Proc. 3rd International Conference on Biomedical Engineering and Informatics (BMEI), pp. 531-535, 2010.

[16] A. Xu, L. Wang, S. Feng, and Y. Qu, "Threshold-based level set method of image segmentation," in Proc. 3rd International Conference on Intelligent Networks and Intelligent Systems (ICINIS), pp. 703-706, 2010.

[17] S. Zhu, X. Xia, Q. Zhang and K. Belloulata, "An Image Segmentation Algorithm in Image Processing Based on Threshold Segmentation", in Proc. Third International IEEE Conference on Signal-Image Technologies and Internet-Based System, SITIS'0., pp. 673-678, 2007.

[18] F. Jiang, M. R. Frater, and M. Pickering, "Threshold-Based Image Segmentation Through an Improved Particle Swarm Optimization", in Proc. International Conference on Digital Image Computing Techniques and Applications (DICTA), pp. 1-5, 2012.

[19] Nameirakpam Dhanachandra, Khumanthem Manglem and Yambem Jina Chanu," Image Segmentation using K-means Clustering Algorithm and Subtractive Clustering Algorithm", Eleventh International Multi-Conference on Information Processing, (IMCIP), pp. $764-771,2015$.

[20] Alan Jose, S. Ravi and M. Sambath, "Brain Tumor Segmentation using K-means Clustering and Fuzzy C-means Algorithm and its Area Calculation", In International Journal of Innovative Research in Computer and Communication Engineering, Vol. 2, No. 2, March 2014.

[21] Pallavi Purohit and Ritesh Joshi, "A New Efficient Approach Towards K-means Clustering Algorithm", In International Journal of Computer Applications, Vol. 65, No. 11, March 2013.

[22] S. Kobashi and J. K. Udupa, "Fuzzy Object Model Based Fuzzy Connectedness Image Segmentation of Newborn Brain MR Images", in Proc. IEEE International Conference on Systems, Man, and Cybernetics (SMC), pp. 1422-1427, 2012.

[23] A. Fabijanska, "Variance Filter for Edge Detection and Edge-Based Image Segmentation", in Proc. International Conference on Perspective Technologies and Methods in MEMS Design (MEMSTECH), pp. 151-154, 2011.

[24] Madhu Yedla, Srinivasa Rao Pathakota and T. M. Srinivasa, "Enhanced K-means Clustering Algorithm with Improved Initial Center", In International Journal of Science and Information Technologies, Vol. 1, No 2, pp. 121-125, 2010.

[25] R. Patil and K. Jondhale, "Edge Based Technique to Estimate Number of Clusters in K-Means Color Image Segmentation", in Proc. 3rd IEEE International Conference on Computer Science and Information Technology (ICCSIT), pp. 117$121,2010$.

[26] L. Yucheng and L. Yubin, "An Algorithm of Image Segmentation Based on Fuzzy Mathematical Morphology", in International Forum on Information Technology and Applications, IFITA'09, pp. 517-520, 2009.

[27] S. A. Ahmed, S. Dey, and K. K. Sarma, "Image Texture Classification using Artificial Neural Network (ANN)", in Proc. 2nd National Conference on Emerging Trends and Applications in Computer Science (NCETACS), pp. 1-4, 2011.

[28] W. Zhao, J. Zhang, P. Li, and Y. Li, "Study of Image Segmentation Algorithm Based On Textural Features and Neural Network", in International Conference on Intelligent Computing and Cognitive Informatics (ICICCI), pp. 300-303, 2010. 
[29] L. Zhang and X. Deng, "The Research of Image Segmentation Based on Improved Neural Network Algorithm", in Proc. Sixth International Conference on Semantics Knowledge and Grid (SKG), pp. 395-397, 2010.

[30] D. Barbosa, T. Dietenbeck, J. Schaerer, J. D'hooge, D. Friboulet, and O. Bernard, "B-Spline Explicit Active Surfaces: An Efficient Framework for Real-Time 3-D Region-Based Segmentation", IEEE Transactions on Image Processing, Vol. 21, pp. 241-251, 2012.

[31] T. Mei, C. Zheng, and S. Zhong, "Hierarchical Region Based Markov Random Field for Image Segmentation", in Proc. International Conference on Remote Sensing, Environment and Transportation Engineering (RSETE), pp. 381-384, 2011.

[32] Z. Hua, Y. Li, and J. Li, "Image Segmentation Algorithm Based on Improved Visual Attention Model and Region Growing", in Proc. 6th International Conference on Wireless Communications Networking and Mobile Computing (WiCOM), pp. 1-4, 2010.

[33] G. Chen, T. Hu, X. Guo, and X. Meng, "A Fast Region-Based Image Segmentation Based on Least Square Method", in Proc. IEEE International Conference on Systems, Man and Cybernetics, SMC, pp. 972-977, 2009.

[34] F. C. Monteiro and A. Campilho, "Watershed Framework to Region-Based Image Segmentation," in Proc. International Conference on Pattern Recognition, ICPR 19th, pp. 1-4, 2008.

[35] M. R. Khokher, A. Ghafoor, and A. M. Siddiqui, "Image Segmentation using Fuzzy Rule Based System and Graph Cuts", in Proc. 12th International Conference on Control Automation Robotics \& Vision (ICARCV), pp. 1148-1153, 2012.

[36] Ping Jiang, Quansheng Dou and Xiaoying Hu, "A Parallel Realization of the Active Contour Model on Boundary Extraction", International Journal of Applied Mathematics \& Information Sciences, Appl. Math. Inf. Sci. Vol. 8, No. 1, pp. 253260, 2014.

[37] A. A. Alexandria, P. C. Cortez, J. A. Bessa, J. H. S. Felix, J. S. Abreu and V. H. Albuquerque, "pSnakes: Anew Radial Active Contour Model and its Application in the Segmentation of the Left Ventricle from Echocardiographic Images", Computer Methods and Programs in Biomedicine, Vol. 116, pp. 260-273, 2014.

[38] John A. Bogovic, Jerry L. Prince and Pierre-Louis Bazin,"Multiple-object Geometric Deformable Model", Computer Vision and Understanding, Vol. 117, pp. 145-157, 2013.

[39] Zhen Yang, John A. Bogovic, Aaron Carass, Mao Ye, Peter C. Searson, Jerry L. Prince, "Automatic Cell Segmentation in Fluorescence Images of Confluent Cell Monolayers Using Multi-Object Geometric Deformable Model", Image Processing, Medical Imaging, Proc. of SPIE, 2013.

[40] Paresh Chandra Barman et.al. "MRI Image Segmentation Using Level Set Method and Implement a Medical Diagnosis System", Computer Science \& Engineering: An International Journal (CSEIJ), Vol.1, No.5, December 2011.

[41] J. Xiao, B. Yi, L. Xu, and H. Xie, "An Image Segmentation Algorithm Based on Level Set Using Discontinue PDE", in Proc. First International Conference on Intelligent Networks and Intelligent Systems, ICINIS'08, pp. 503-506, 2008.

[42] Kabade Mankarnika Manohar and A. S. Patil, "A Review on Techniques of Image Segmentation", International Journal of Advanced Research in Computer and Communication Engineering (IJARCCE), Vol. 5, No. 3, March 2016. 
[43] Sonam Mandiratta, Pooja Batra Nagpal and Sarika Chaudhary, "A Perlustration of Various Image Segmentation Techniques", International Journal of Computer Applications Vol. 139, No. 12, April 2016.

[44] Aman Mittal, "A Review on Image Segmentation Techniques", International Journal of Recent Advances in Engineering \& Technology (IJRAET), Vol. 4, No. $1,2016$.

[45] Arti Taneja, Priya Ranjan and Amit Ujjlayan, "A Performance Study of Image Segmentation Techniques", IEEE, 2015.

[46] Amanpreetkaur and Navjotkaur, "Image Segmentation Techniques", International Research Journal of Engineering and Technology (IRJET), Vol. 2, No. 2, 2015.

[47] Priyanka Shivhare and Vinay Gupta, "Review of Image Segmentation Techniques Including Pre \& Post Processing Operations", International Journal of Engineering and Advanced Technology (IJEAT), Vol. 4, No. 3, 2015.

[48] Pooja Rani and Rakesh Verma,"A Review on Ultrasound Image Segmentation Techniques", International Journal of Advanced Research in Electronics and Communication Engineering (IJARECE), Vol. 4, No. 8, 2015.

[49] Muhammad Waseem Khan, "A Survey: Image Segmentation Techniques", International Journal of Future Computer and Communication, Vol. 3, No. 2, 2014.

[50] M. Jogendra Kumar, Dr. GVS Raj Kumar and R. Vijay Kumar Reddy, "Review on Image Segmentation Techniques", International Journal of Scientific Research Engineering \& Technology (IJSRET), Vol. 3, No. 6, 2014.

[51] Rohan Kandwal, Ashok Kumar and Sanjay Bhargava, "Review: Existing Image Segmentation Techniques", International Journal of Advanced Research in Computer Science and Software Engineering, Vol. 4, No. 4, 2014.

[52] Anamika Ahirwar, "Study of Techniques used for Medical Image Segmentation and Computation of Statistical Test for Region Classification of Brain MRI", International Journal of Information Technology and Computer Science, pp. 4453, April 2013.

[53] Cristina S. and Ştefan H., "A Comparison of X-Ray Image Segmentation Techniques", Advances in Electrical and Computer Engineering, Vol. 13, No. 3, 2013.

[54] Ajala Funmilola A et. al.,"Fuzzy k-c-means Clustering Algorithm for Medical Image Segmentation", Journal of Information Engineering and Applications, Vol 2, No.6, 2012.

[55] Krishna Kant Singh and Akansha Singh,"A study of Image Segmentation Algorithms for Different Types of Images", International Journal of Computer Science Issues (IJCSI), Vol. 7, No. 5, September 2010.

[56] https://omictools.com/image-segmentation-category (Access date, August 2016). 
تخفيض الضبابية والتقبيم الكفء لصور الموجات فوق الصوتية

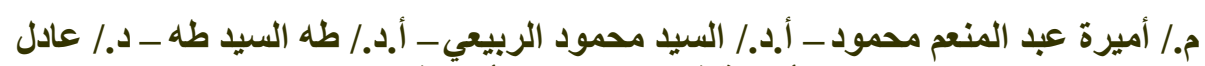

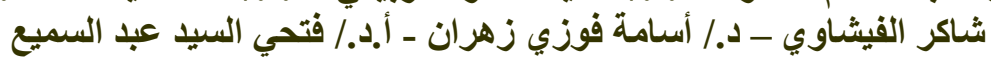

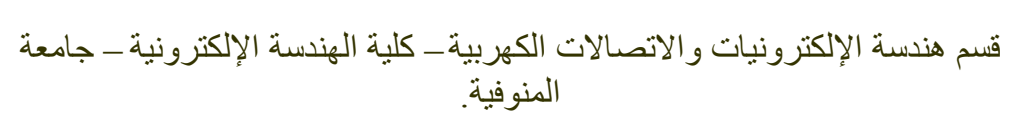

يتطلب التقسيم فصل أو تقطيع الصورة إلى مناطق ذات خصائص متماثلة.

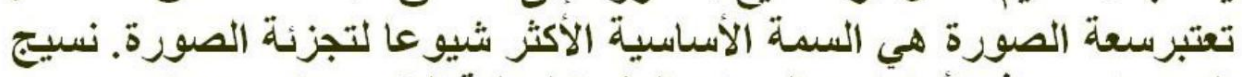

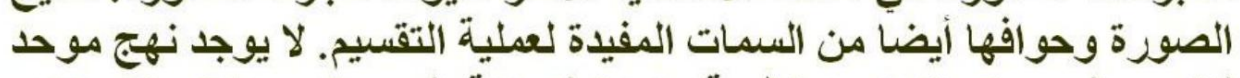

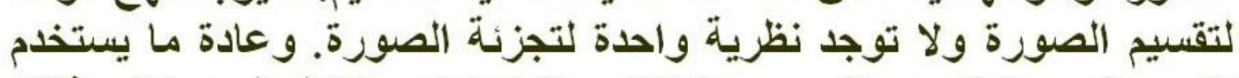

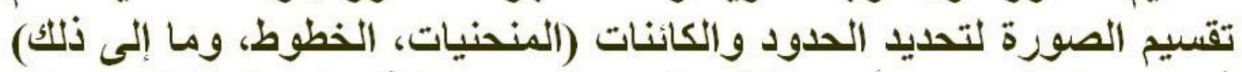

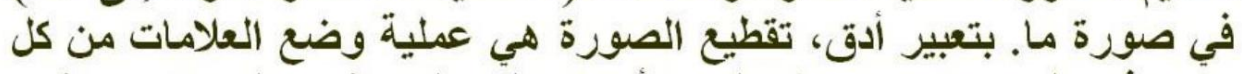

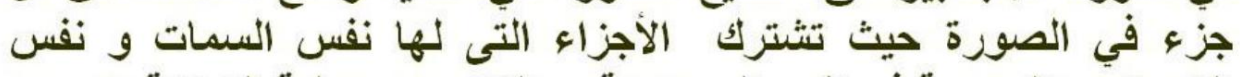

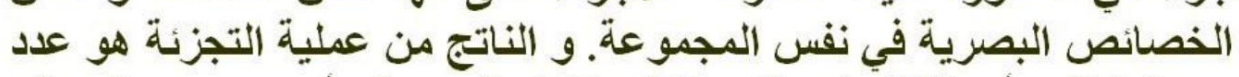

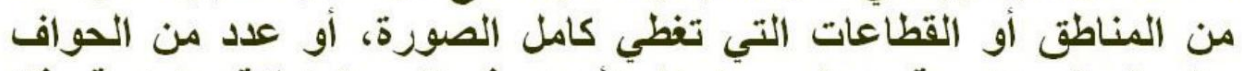

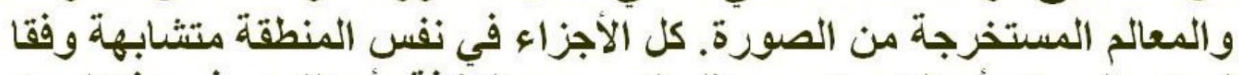

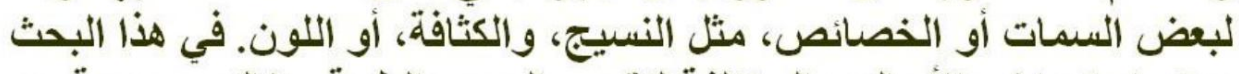

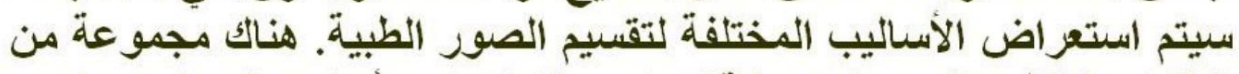

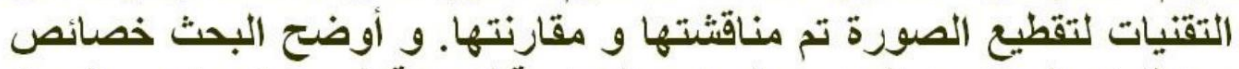

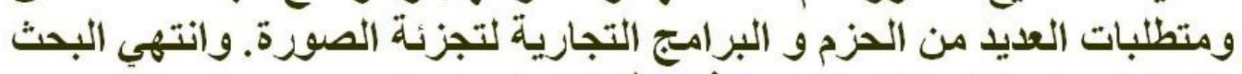

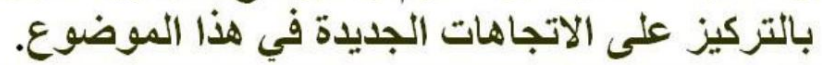

